\title{
Influence of Particle Size Distribution on Random Close Packing of Spheres
}

\author{
Kenneth W. Desmond and Eric R. Weeks \\ Department of Physics, Emory University, Atlanta, Georgia 30322, USA
}

(Dated: June 24, 2013)

\begin{abstract}
The densest amorphous packing of rigid particles is known as random close packing. It has long been appreciated that higher densities are achieved by using collections of particles with a variety of sizes. For spheres, the variety of sizes is often quantified by the polydispersity of the particle size distribution: the standard deviation of the radius divided by the mean radius. Several prior studies quantified the increase of the packing density as a function of polydispersity. Of course, a particle size distribution is also characterized by its skewness, kurtosis, and higher moments, but the influence of these parameters has not been carefully quantified before. In this work, we numerically generate many sphere packings with different particle radii distributions, varying polydispersity and skewness independently of one another. We find two significant results. First, the skewness can have a significant effect on the packing density and in some cases can have a larger effect than polydispersity. Second, the packing fraction is relatively insensitive to the value of the kurtosis. We present a simple empirical formula for the value of the random close packing density as a function of polydispersity and skewness.
\end{abstract}

\section{INTRODUCTION}

Understanding various aspects of random close packing $(r c p)$ has great scientific and industrial importance [1 as it has been linked to a wide range of problems such as the structure of living cells [2, liquids [3, 4, granular media [5] 8, emulsions 9, glasses [10, amorphous solids [11, jamming [12, 13, the viscosity of suspensions [14, 15], and the processing of ceramic materials [16. Random close packing is typically defined as a collection of particles packed into the densest possible amorphous configuration, although more rigorous definitions are available [17. Experiments have found that the densest random packing of monodisperse spheres typically occurs close to $\phi_{0, r c p} \sim 0.64$ [4, where the density $\phi$ (or packing fraction) is defined as the ratio of the total volume occupied by spheres to the volume of the container.

Formally, a packing consists of particles with a distribution in radii $P(R)$. The polydispersity is defined as

$$
\delta=\sqrt{\left\langle\Delta R^{2}\right\rangle} /\langle R\rangle .
$$

Here, $\Delta R=R-\langle R\rangle$ and the moments of $R$ (and $\Delta R$ ) are defined as $\left\langle R^{n}\right\rangle=\int R^{n} P(R) d R$ [and $\left\langle\Delta R^{n}\right\rangle=$ $\left.\int \Delta R^{n} P(R) d R\right]$. It has long been appreciated that packings of spheres can have larger $r c p$ densities when $\delta>0$ [18 23]. Prior experiments [24 28 and simulations 29 33 have nicely shown that as the polydispersity increases, the particles pack to higher volume fractions because the smaller particles pack more efficiently by either layering against larger particles or by fitting into the voids created between neighboring large particles 25, 34 36. In practice, depending on the degree of the polydispersity, the packing fraction can increase from 0.64 for monodisperse packings to nearly $\sim 0.75$ for packings with 0.65 polydispersity 25]. For the extreme case of two different particle sizes with a size ratio approaching infinity, the voids between the large particles can be packed randomly with small particles and so $\phi$ can be as large as $\phi_{0, r c p}+\left(1-\phi_{0, r c p}\right) \phi_{0, r c p} \approx 0.88$ [36, 37.
While it is intuitive that the polydispersity can affect $\phi_{r c p}$, it is also reasonable that the shape, not just the spread, of the distribution $P(R)$ may also influence $\phi_{r c p}$ [1, 25. For instance, an infinite number of distributions can have the same value of $\delta$ but yet differ in their form. One can characterize the shape using the skewness

$$
S=\left\langle\Delta R^{3}\right\rangle /\left\langle\Delta R^{2}\right\rangle^{3 / 2},
$$

kurtosis

$$
K=\left\langle\Delta R^{4}\right\rangle /\left\langle\Delta R^{2}\right\rangle^{2},
$$

and higher moments. There have been prior studies that have investigated the influence of distribution shape on the density of tightly packed particles [16, 25, 36, 38, 42. Similar to the studies on polydispersity, they find that the shape of the particle distribution can have a profound influence on the packing density. However, these prior studies either did not independently vary $\delta$ and $S$ but rather conflated the influences of both, or else used other metrics besides $\delta$ and $S$ to quantify $P(R)$. Of the prior studies, Tickell et al. [43] is the only one to report on the effects of skewness and kurtosis for experiments carried out with sand, finding that over a narrow range in skewness the packing density can increase by 0.04 with no dependence on kurtosis. However, they did not control for polydispersity, leaving it unclear the relative importance of polydispersity and skewness. The key unanswered question by the prior work is how the skewness of a distribution influences $\phi_{r c p}$, and how large this effect is relative to the effects of polydispersity.

In this paper, we address this question by numerically generating packings with a variety of particle size distributions. We find that both polydispersity $\delta$ and skewness $S$ influence the maximum random close packing volume fraction. In particular, increasing $\delta$ increases $\phi_{r c p}$, and for a given $\delta, \phi_{r c p}$ increases linearly with increasing $S$. As $S$ can be negative, a negatively skewed $P(R)$ can decrease $\phi_{r c p}$ as compared to a symmetric distribution. We find no universal influence of the kurtosis on our results. 


\section{PROTOCOL}

We need to generate packings with arbitrary size distributions $P(R)$, with a goal of controlling $\delta$ and $S$ independently. Our method for generating these packings was previously developed in Ref. 44. Briefly, infinitesimal particles are placed randomly in a periodic container, gradually expanded, and moved at each step to prevent particles from overlapping. At the beginning of the simulation, particles are assigned radii with a specific distribution and as the particles expand they do so by a multiplicative factor such that the shape of the radii distribution is fixed. The value of $\phi_{r c p}$ is known to be sensitive to protocol [45, 46], and it is not known if this algorithm or any other algorithm produces rigorously defined random close packed states [17, 44, 47, 48]. Our goal is not to determine the precise value of $\phi_{r c p}$ for a given $P(R)$, but rather to empirically understand the trend in $\phi_{\text {rcp }}$ with polydispersity and skewness. Happily, our algorithm when applied to a monodisperse packing gives $\phi_{0, r c p} \sim 0.64$, close to the experimentally found value and in agreement with prior simulation work. In practice, the simulation has three adjustable parameters that determine how quickly the simulation converges to a rcp state. These parameters are the initial packing fraction, the rate of expansion/contraction, and a threshold on the minimum energy (see Ref. [49] for more details). We use the same values as in Ref. [49], and we find that our algorithm produces reproducible results and $\phi_{r c p}$ is not sensitive to slight changes in these values.

To efficiently determine $\phi_{r c p}$ for a chosen particle size distribution, we exploit the known finite size dependence $\phi_{r c p}(h)=\phi_{r c p}^{\infty}-C / h$, where $h$ is the system size, $\phi_{r c p}^{\infty}$ is the random close packing fraction in the limit $h \rightarrow \infty$, and $C$ is a fitting constant [49]. By generating many packings with different periodic box sizes $h$, we fit $\phi_{r c p}(h)$ to determine $\phi_{r c p}^{\infty}$ for each distribution. We generate packings with box sizes of $\sim 10,14,18$, and 23 mean particle diameters in length to determine $\phi_{r c p}^{\infty}$. For the rest of the paper, $\phi_{r c p}$ will be used to indicate $\phi_{r c p}^{\infty}$.

To control for both $\delta$ and $S$ independently, we study packings using four different distributions: binary, linear, gaussian, and lognormal. The binary and linear distributions are determined by two control parameters, allowing for us to control $\delta$ and $S$ independently, while the gaussian and lognormal distributions are determined by only one parameter, and therefore $\delta$ and $S$ can not be controlled independently. By generating many packings with different $\delta$ and $S$ using these four distributions, we can compare the results to see how sensitive $\phi_{r c p}$ is to polydispersity and skewness, but we can also compare different distributions with the same $\delta$ and $S$ to see how sensitive $\phi_{r c p}$ is to other subtle differences in the distribution shape. For all distributions, we impose $\langle R\rangle=1$.

More specifically, the binary distribution consists of particles with two distinct radii. The shape of the distribution is determined by the size ratio and number ratio of these two particle types. The linear distribution is a con-

\begin{tabular}{|c|c|}
\hline Binary & \\
\hline Function & $P(R)=(1-\rho) \delta(R-a)+\rho \delta(R-b)$ \\
\hline Parameters & Number ratio $\rho=P(b) / P(a)$ \\
\hline & Size ratio $\eta=b / a$ \\
\hline Constrained & $a=1 /(1-\rho+\eta \rho)$ \\
\hline & $b=\eta /(1-\rho+\eta \rho)$ \\
\hline Polydispersity & $\delta=\left((1-\rho)(a-1)^{2}+\rho(b-1)^{2}\right)^{1 / 2}$ \\
\hline Skewness & $\left((1-\rho)(a-1)^{3}+\rho(b-1)^{3}\right) / \delta^{3}$ \\
\hline Kurtosis & $\left((1-\rho)(a-1)^{4}+\rho(b-1)^{4}\right) / \delta^{4}$ \\
\hline Linear & \\
\hline Function & $P(R)=A R+B, a \leq R \leq b$ \\
\hline Parameters & $\rho=P(b) / P(a)$ \\
\hline & $\eta=b / a$ \\
\hline Constrained & $a=3(1+\rho)(\eta-1) /(4-\rho)$ \\
\hline & $b=3 \eta(1+\rho)(\eta-1) /(4-\rho)$ \\
\hline & $A=2(\rho-1)(4-\rho) /\left(9(1+\rho)^{3}(\eta-1)^{4}\right)$ \\
\hline & $B=2(2-\rho)(4-\rho) /\left(3(1+\rho)^{2}(\eta-1)^{3}\right)$ \\
\hline Polydispersity & Solved numerically \\
\hline Skewness & Solved numerically \\
\hline Kurtosis & Solved numerically \\
\hline Gaussian & \\
\hline Function & $P(R)=A e^{-(R-1)^{2} / 2 \sigma^{2}}$ \\
\hline Parameters & Standard deviation $\sigma$ \\
\hline Constrained & $A=1 / \sqrt{2 \pi \sigma}$ \\
\hline Polydispersity & $\sigma$ \\
\hline Skewness & 0 \\
\hline Kurtosis & 0 \\
\hline Lognormal & \\
\hline Function & $P(R)=\frac{A}{R} e^{\left.(\ln R / \sigma+0.5 \sigma)^{2}\right) / 2}$ \\
\hline Parameters & Scale parameter $\sigma$ \\
\hline Constrained & $A=1 / \sqrt{2 \pi \sigma}$ \\
\hline Polydispersity & $e^{\sigma} \sqrt{e^{\sigma^{2}}-1}$ \\
\hline Skewness & $\left(e^{\sigma^{2}}+2\right) \sqrt{e^{\sigma^{2}}-1}$ \\
\hline Kurtosis & $e^{4 \sigma^{2}}+2 e^{3 \sigma^{2}}+3 e^{2 \sigma^{2}}-6$ \\
\hline
\end{tabular}

TABLE I. This table summarizes the distributions applied in this study. The first row indicates the functional form of the distribution. The shape of $P(R)$ is controlled by some free parameters indicated in the next row. Each distribution is constrained such that the mean particle radius is unity and the probability to find any particle size is unity, which constrain some of the coefficients in $P(R)$ to fixed values. The row titled "Constrained" lists the fixed values of these coefficients. The last rows list the polydispersity. skewness, and kurtosis for the distribution. For the binary distribution, note that $\delta(R)$ is the Dirac delta function. For the linear distribution, analytic solutions are unnecessarily long for the polydispersity. skewness, and kurtosis and were computed numerically for simplicity. For the Gaussian and lognormal distributions, a truncation was applied to ensure that no particle radii were below 0.1 . This affects the shape slightly, and the polydispersity. skewness, and kurtosis were computed numerically when truncation was applied. 


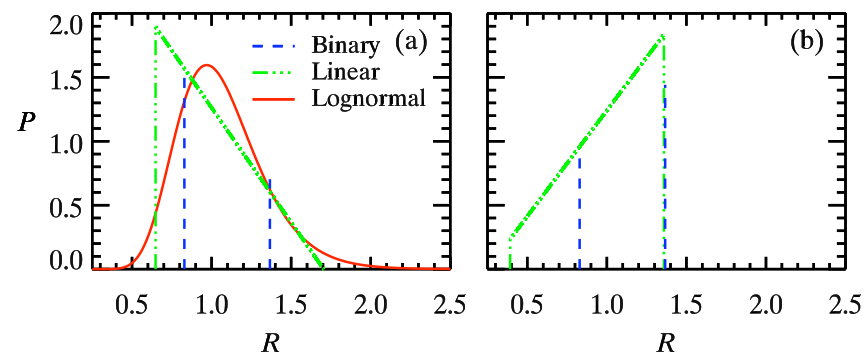

FIG. 1. (Color online). (a) Examples of three different particle radii distributions with the same polydispersity of 0.25 and nearly the positive same skewness. The binary distribution has $S=0.78$, the linear distribution has $S=0.56$, and the lognormal distribution has $S=0.78$. (b) Examples of two different particle radii distributions with the same polydispersity of 0.25 and negative skewness $S=-0.5$.

tinuous distribution of the form $P(R)=A R+B$, where the distribution in particle size exists over a finite range $a \leq R \leq b$. Our choice of $\langle R\rangle=1$ and the requirement of normalization $\left(\int_{a}^{b} P(R) d R=1\right)$ imposes two constraints on the parameters $(a, b, A, B)$. For the two remaining degrees of freedom, we define $\eta=b / a$ and $\rho=P(a) / P(b)$. We compute $S$ and $\delta$ for a grid of $\eta$ and $\rho$ values, and then interpolate to find the parameters for $P(R)$ for the desired $S$ and $\delta$ values, allowing us to vary them systematically. The third distribution is a Gaussian of the form $P(R)=A_{G} \exp \left(-(R-1)^{2} / 2 \sigma^{2}\right)$, where $\sigma$ is the standard deviation and $A_{G}=1 /(\sigma \sqrt{2 \pi})$. For larger $\sigma$, some of the particle radii could be negative, which is unphysical, or very close to zero, which may prevent generating packings within a reasonable time frame. To avoid these issues, we truncate the Gaussian distribution such that the smallest particle radius is no smaller than 0.1. The Gaussian distribution has a fixed skewness $S=0$ except for the truncated Gaussians, which have a slight positive skewness. The last distribution we consider is the lognormal distribution $P(R)=A_{L} \exp \left(-0.5(\ln R / \sigma+0.5 \sigma)^{2}\right) / R$, where $A_{L}=1 /(\sigma \sqrt{2 \pi})$. Similar to the Gaussian distribution, the skewness of the lognormal distribution is not adjustable, but is always positive and becomes larger as $\sigma$ becomes larger. We provide a summary of the distributions in Table \.

\section{RESULTS \& DISCUSSION}

In Fig. 1(a), we compare three different distributions with polydispersity $\delta=0.25$ and nearly the same positive skewness $S \approx 0.75$. We see that the distributions are quite different, in particular in their tails. For example, the linear distribution has many more small particles than the other two distributions. The lognormal distribution has tails that include both smaller and larger particles than the other two distributions. It's not necessarily obvious how the values of $\phi_{r c p}$ will be ranked for these

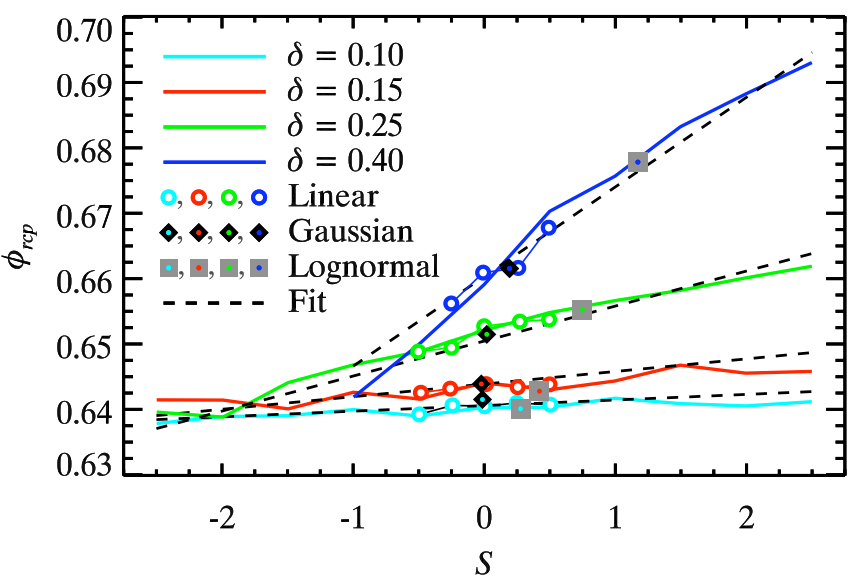

FIG. 2. (Color online). This figure shows how $\phi_{r c p}$ depends on particle size distribution, polydispersity $\delta$, and skewness $S$. The solid lines represent $\phi_{r c p}$ for binary packings and the symbols represent $\phi_{r c p}$ for packings with either linear, Gaussian, or lognormal particle distributions as indicated by the legend. The colors represent different polydispersities of either $0.1,0.15,0.25$, or 0.4 . The dashed lines are a fit to the data using $\phi_{r c p}=\phi_{r c p}^{*}+c_{1} \delta+c_{2} S \delta^{2}$, where $\phi_{r c p}^{*}=0.634$, $c_{1}=0.0658$, and $c_{2}=0.857$.

cases. In Fig. 1 (b), we show two different distributions with polydispersity $\delta=0.25$ and skewness $S \approx-0.5$. As these distributions have negative skewness, both distributions have more larger particles than smaller particles. Once again, it's not necessarily clear how $\phi_{r c p}$ should differ between the two packings.

After generating nearly 10,000 packings with different particle radii distributions, we plot $\phi_{r c p}$ as a function of skewness $S$ for all our data in Fig. 2, with the different groups of data (different colors) corresponding to different polydispersity values $\delta$. Each data point in the figure has a one to one correspondence to the distribution type, $\delta$, and $S$. The symbol or line type of the data indicates the $P(R)$ distribution type. Remarkably, the figure shows that regardless of the type of particle radii distribution, $\phi_{r c p}$ is nearly the same for the same pairing of polydispersity and skewness. It also shows that $\phi_{r c p}$ increases with both increasing $\delta$ and $S$. Strikingly, the skewness can have an equally important effect as the polydispersity. For example, for $\delta=0.40$ and $S=0, \phi_{r c p}$ is shifted upward by $\approx 0.02$. Fixing that value of $\delta$, changing $S$ to \pm 1 shifts $\phi_{r c p}$ by $\approx \pm 0.02$. For highly polydisperse samples, one cannot accurately know $\phi_{r c p}$ without also knowing the skewness of the radius distribution. For the binary samples (solid lines in Fig. 2) $S$ can be even larger in magnitude and have an even larger influence on $\phi_{r c p}$ than $\delta$ has.

The increase in $\phi_{r c p}$ with skewness is not uniform. For negative skewness (more big particles), the polydispersity $\delta$ does not seem to influence $\phi_{r c p}$ as much as when the skewness is positive. This is not too surprising since the volume of each particle grows with $R^{3}$. When the 


$$
\begin{aligned}
& \text { Lognormal } \\
& \delta=0.106, S=0.440 \\
& \phi=0.640
\end{aligned}
$$
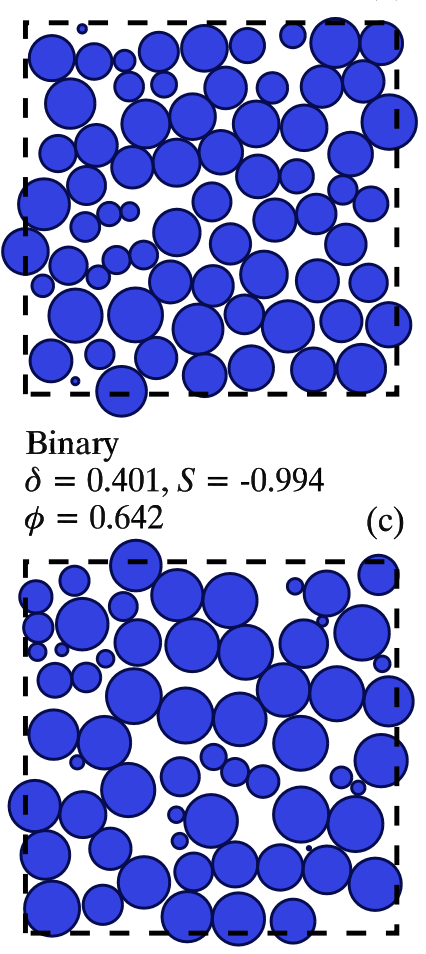

(c)

(a)
Lognormal

$\delta=0.424, S=1.36$

$\phi=0.678$

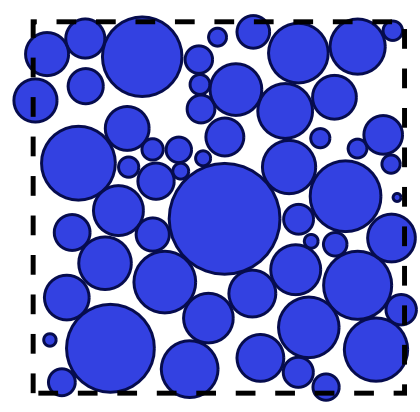

Binary

$\delta=0.400, S=2.00$

$\phi=0.688$

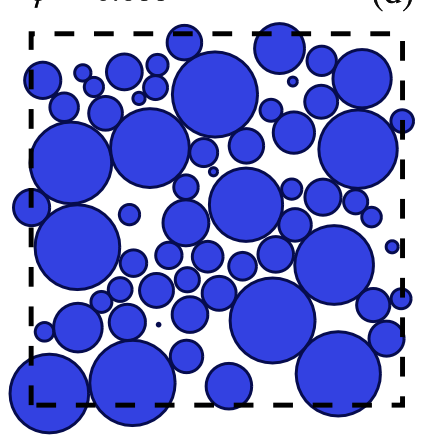

FIG. 3. (Color online). Each image represents a $2 \mathrm{D}$ slice through a $3 \mathrm{D}$ packing, and the dashed box is the boundary of the periodic packing. The volume fraction for each packing shown is close to the extrapolated $\phi_{r c p}$. Also, the area fraction of each $2 \mathrm{D}$ slice is the same as the volume fraction of the $3 \mathrm{D}$ packing they represent.

total number of bigger particles is greater than the total number of smaller particles (negative skewness), the volume occupied by all the large particles is significantly greater than the volume occupied by all the small particles. In effect, the big particles pack like a low polydispersity sample and occupy the majority of the container, while the small spheres occupy an insignificant portion, and $\phi_{r c p}$ approaches $\phi_{0, r c p} \approx 0.64$ for a monodisperse sample. For positive skewness (more smaller particles), $\phi_{\text {rcp }}$ has a fairly strong dependence on $\delta$ and $S$, where $\phi_{r c p}$ increases with increasing number of small particles. The reason for this increase in $\phi_{r c p}$ is likely due to the small particles fitting into the spaces between larger particles. As discussed in prior work [25, 35, 36, the local porosity is smaller around two neighboring particles of different sizes than around two neighboring particles of the same size. This effect is greater for larger differences in the size of two neighbors. As skewness and polydispersity increase, both the number of small particles present and the average size discrepancy between neighboring particles increase, resulting in a larger $\phi_{r c p}$.

To provide a qualitative sense of the behavior, Fig. 3

shows a 2D slice through four different 3D packings. (a) and (b) are lognormal packings, where (a) is a packing at low polydispersity and skewness and (b) is a denser packing at a higher polydispersity and skewness. Packings (c) and (d) are two binary packings with polydispersity 0.4 , where (c) has a large negative skewness and (d) is denser and has a large positive skewness. As discussed above, at large $\delta$ and $S$, small particles can either layer around larger particles and/or fit in the voids between bigger particles. In Fig. 3(b) and (d) we see evidence of small particles sitting in the void areas between big particles. In Fig. 3(a) and (c), where the skewness is lower, we see less evidence of this. These observations are consistent with the results of Fig. 2.

Since $\phi_{r c p}$ is nearly determined by the two parameters $\delta$ and $S$, we fit all the data to a simple equation

$$
\phi_{r c p}=\phi_{r c p}^{*}+c_{1} \delta+c_{2} S \delta^{2},
$$

where $\phi_{r c p}^{*}=0.634$ is the packing fraction for a monodisperse packing of spheres $(\delta=0$ and $S=0)$ and $c_{1}=$ 0.0658 and $c_{2}=0.857$ are empirical constants. These fit lines are shown as dashed lines in Fig. 2, and agree reasonably well with the data. Our fitted value of $\phi_{r c p}^{*}$ is close to the experimentally accepted value of $0.637[1,50$. We also tried fits with higher order terms in $S$ and $\delta$, but we found first order in $S$ and second order in $\delta$ reasonably fit all the data well.

There are slight differences in the $\phi_{r c p}$ values for different distribution types for the same $\delta$ and $S$ values, seen in Fig. 2. Thus far we have focused on $\delta$ and $S$ to characterize our distributions, and of course the distributions differ in their higher moments. The next quantity to consider is the kurtosis $K$ defined above (Eqn. 3), and it might be a potential additional parameter to explain the variations in Fig. 2. To check this, we subtract the computationally found values of $\phi_{r c p}$ from the empirical fit (Eqn. 4) and plot these differences as a function of $K$ (not shown), which shows no systematic dependence on $K$. That is, $K$ seems not to be a useful fit parameter for $\phi_{r c p}$. This agrees with the 1933 qualitative observations of Tickell et al. 43 .

It is worth noting that our results apply to randomly packed objects, and one can consider other amorphous packings that are perhaps less random. For example, an important class of these are random Apollonian packings (RAP) [51, 52. To generate a RAP, one first places the largest spheres, then smaller particles are inserted into the voids between the spheres. For example, one can fill the voids with the largest possible spheres, [51, which leads to a packing with a volume fraction approaching arbitrarily close to 1.0 . In such situations, $P(R)$ is a power law, $P(R) \sim R^{-\alpha}$ for $R>R_{0}$, where $R_{0}$ is a cutoff. As $R_{0}$ approaches 0 (an infinite amount of iterations of the RAP protocol), the volume fraction approaches 1.0. Using our equations, you can see that if $R_{0}=0$, $\left\langle R^{n}\right\rangle$ is infinite for $\left.n\right\rangle=\alpha-1$. For finite but small $R_{0}$, $\left\langle r^{n}\right\rangle$ can be quite large, and thus the packing can have large values for $\delta, S$, and $K$. Clearly in such limits the 
volume fraction nonetheless is 1.0 or smaller, so our empirical formula Eqn. 4 must break down. On the other hand, the RAP protocols all ensure large volume fractions by construction - that is, the small particles are precisely chosen to fit into the voids between the large ones, and $P(R)$ is determined after the fact through the algorithm. Our computational algorithm will generally find less optimal packings for the same $P(R)$, and so it is to be expected that Eqn. 4 should not apply to RAP.

For that matter, our algorithm converges unacceptably slowly for distributions with particle sizes varying by more than a factor of ten between the smallest and largest sizes, preventing us from directly testing power law packings. We work around this by using the numerical algorithm proposed by Farr and Groot 37, which rapidly predicts $\phi_{r c p}$ based on any $P(R)$ as input. The predictions of their algorithm agree well with the results from our computed 3D packings for the distributions listed in Table I. We use their algorithm to determine $\phi_{r c p}$ for a variety of power law distributions over the same range of $\delta$ and $S$ tested for our other distributions, and we find excellent agreement with our empirical expression Eqn.4.

\section{CONCLUSIONS}

Our data have two significant conclusions. First, the skewness $S$ has a significant influence on $\phi_{r c p}$ for distribu- tions with a large polydispersity $\delta$. Second, Eqn. 4 allows one to determine $\phi_{r c p}$ to within approximately \pm 0.002 from knowing $\delta$ and $S$, without taking into account any other details of the shape of $P(R)$.

This collapse of $\phi_{r c p}$ values for a given $\delta$ and $S$ but different distribution shapes is intriguing, as presumably the structures within the packings are different for different $P(R)$. For that matter, one can have the same $\phi_{r c p}$ value for different $\delta$ and $S$, see for example Fig. 3(a) and (c), and clearly these will have different microstructures. This might be useful for studying aspects of the jamming transition of spherical particles. Many prior results show that various properties of these systems depend on the distance to the jamming point [53 57, where the jamming point is thought to be the same as $\phi_{\text {rcp }}$ [17, 47, 48, 58]. One can imagine conducting experiments or simulations to compare the properties of packings near the jamming transition with different microstructures, but the same jamming point. These could be equally useful for studying the colloidal glass transition, which may be influenced by $\phi_{r c p}[55,5963$. Such experiments may provide further insight into the universal nature of the jamming transition and glass transition, but may also highlight subtle dependencies on the microstructure.

The work of K.W.D was supported by the National Science Foundation under Grant No. CBET-0853837, and the work of E.R.W. was supported by the National Science Foundation under Grant No. CMMI-1250235.
[1] S. Torquato and F. H. Stillinger, Rev. Mod. Phys. 82, 2633 (Sep. 2010)

[2] T. M. Truskett, S. Torquato, and P. G. Debenedetti, Phys. Rev. E 62, 993 (2000)

[3] O. K. Rice, J. Chem. Phys. 12, 1 (1944)

[4] J. D. Bernal and J. Mason, Nature 188, 910 (1960)

[5] W. O. Smith, P. D. Foote, and P. F. Busang, Phys. Rev. Lett. 34, 1271 (1929)

[6] S. F. Edwards, Granular Matter (Springer-Verlag, 1994)

[7] C. Radin, J. Stat. Phys. 131, 567 (2008)

[8] M. Jerkins, M. Schröter, H. L. Swinney, T. J. Senden, M. Saadatfar, and T. Aste, Phys. Rev. Lett. 101, 018301 (2008)

[9] R. Pal, Polym. Eng. Sci. 48, 1250 (2008)

[10] G. Lois, J. Blawzdziewicz, and C. S. O'Hern, Phys. Rev. Lett. 102, 015702 (2009)

[11] R. Zallen, Physics of Amorphous Solids (Wiley, 1983)

[12] C. S. O'Hern, L. E. Silbert, A. J. Liu, and S. R. Nagel, Phys. Rev. E 68, 011306 (2003)

[13] C. S. O'Hern, L. E. Silbert, A. J. Liu, and S. R. Nagel, Phys. Rev. E 70, 043302 (2004)

[14] I. M. Krieger and T. J. Dougherty, Transactions of the Society of Rheology 3, 137 (1959), http://dx.doi.org/ 10.1122/1.548848

[15] A. Dörr, A. Sadiki, and A. Mehdizadeh, Journal of Rheology 57, 743 (2013), ISSN 01486055, http://dx.doi. org/10.1122/1.4795746

[16] R. K. Mcgeary, J. Am. Ceram. Soc. 44, 513 (1961)
[17] S. Torquato, T. M. Truskett, and P. G. Debenedetti, Phys. Rev. Lett. 84, 2064 (2000)

[18] A. R. Kansal, S. Torquato, and F. H. Stillinger, J. Chem. Phys. 117, 8212 (2002)

[19] R. Al-Raoush and M. Alsaleh, Powder Technol. 176, 47 (2007)

[20] K. Lochmann, L. Oger, and D. Stoyan, Solid State Sci. 8, 1397 (2006)

[21] H. J. H. Brouwers, Phys. Rev. E 74, 031309 (Sep. 2006)

[22] T. Okubo and T. Odagaki, J. Phys-Condens. Mat. 16, 6651 (2004)

[23] P. Richard, L. Oger, J. P. Troadec, and A. Gervois, Euro. Phys. J. E 6, 295 (Dec. 2001)

[24] B. R. Aïm and L. P. Goff, Powder Technol. 1, 281 (1967)

[25] H. Y. Sohn and C. Moreland, Can. J. Chem. Eng. 46, 162 (1968)

[26] A. R. Dexter and D. W. Tanner, Science 238, 31 (1972)

[27] W. M. Visscher and M. Bolsterli, Nature 239, 504 (1972)

[28] D. M. E. Thies-Weesie and A. P. Philipse, Journal of Colloid and Interface Science 162, 470 (1994)

[29] A. Donev, S. Torquato, F. H. Stillinger, and R. Connelly, J. App. Phys. 95, 989 (2004)

[30] A. Donev, F. H. Stillinger, and S. Torquato, Phys. Rev. Lett. 96, 225502 (Jun. 2006)

[31] T. S. Hudson and P. Harrowell, J. Phys. Chem. B 112, 8139 (Jul. 2008)

[32] W. Schaertl and H. Sillescu, J. Stat. Phys. 77, 1007 (1994) 
[33] M. Hermes and M. Dijkstra, Europhys. Lett. 89, 38005 (2010)

[34] M. Clusel, E. I. Corwin, A. O. N. Siemens, and J. Brujic, Nature 460, 611 (2009)

[35] H. J. H. Brouwers, Phys. Rev. E 74, 031309 (2006)

[36] C. C. Furnas, Ind. Eng. Chem. 23, 1052 (1931)

[37] R. S. Farr and R. D. Groot, J. Chem. Phys. 131, 244104 (2009)

[38] D. P. Haughey and G. S. G. Beveridge, Can. J. Chem. Eng. 47, 130 (1969)

[39] M. Subbanna, P. C. Kapur, and Pradip, Ceram. Int. 28, 401 (Jan. 2002)

[40] M. Suzuki and T. Oshima, Powder Technol. 44, 213 (Oct. 1985)

[41] M. J. Powell, Powder Technol. 25, 45 (Jan. 1980)

[42] H. D. Lewis and A. Goldman, J. Am. Ceram. Soc. 49, 323 (1966)

[43] F. G. Tickell, O. E. Mechem, and R. C. McCurdy, T. Am. I. Min. Met. Eng. 103, 250 (1933)

[44] N. Xu, J. Blawzdziewicz, and C. S. O'Hern, Phys. Rev. E 71, 061306 (2005)

[45] W. S. Jodrey and E. M. Tory, Phys. Rev. A 32, 2347 (1985)

[46] J. Tobochnik and P. M. Chapin, J. Chem. Phys. 88, 5824 (1988)

[47] A. Donev, S. Torquato, F. H. Stillinger, and R. Connelly, Phys. Rev. E 70, 043301 (2004)

[48] C. S. O'Hern, L. E. Silbert, A. J. Liu, and S. R. Nagel, Phys. Rev. E 70, 043302 (2004)

[49] K. W. Desmond and E. R. Weeks, Phys. Rev. E 80, 051305 (2009)
[50] G. D. Scott and D. M. Kilgour, J. Phys. D Appl. Phys. 2, 863 (1969)

[51] G. W. Delaney, S. Hutzler, and T. Aste, Phys. Rev. Lett. 101, 120602 (Sep. 2008)

[52] S. D. S. Reis, N. A. M. Araújo, A. Jr, and H. J. Herrmann, Europhys. Lett., 18004(Jan. 2012)

[53] W. G. Ellenbroek, E. Somfai, M. van Hecke, and W. van Saarloos, Phys. Rev. Lett. 97, 258001 (2006)

[54] T. S. Majmudar, M. Sperl, S. Luding, and R. P. Behringer, Phys. Rev. Lett. 98, 058001 (2007)

[55] A. J. Liu and S. R. Nagel, Condens. Matter Phys. 1, 347 (2010)

[56] K. W. Desmond, P. J. Young, D. Chen, and E. R. Weeks, Soft Matter 9, 3424 (2013)

[57] G. Katgert and M. van Hecke, Europhys. Lett. 34002(2010)

[58] C. S. O'Hern, L. E. Silbert, A. J. Liu, and S. R. Nagel, Phys. Rev. E 68, 011306 (2003)

[59] L. Berthier and G. Biroli, Rev. of Mod. Phys. 83, 587 (Jun. 2011)

[60] P. Chaudhuri, L. Berthier, and W. Kob, Phys. Rev. Lett. 99, 060604 (Aug. 2007)

[61] G. L. Hunter and E. R. Weeks, Rep. Prog. Phys. 75, 066501 (Jun. 2012)

[62] E. Marcotte, F. H. Stillinger, and S. Torquato, J. Chem. Phys. 138, 12 A508 (2013)

[63] Z. Zhang, N. Xu, D. T. N. Chen, P. Yunker, A. M. Alsayed, K. B. Aptowicz, P. Habdas, A. J. Liu, S. R. Nagel, and A. G. Yodh, Nature 459, 230 (2009) 